\title{
Cloud Service Selection using DEA based on SMI Attributes
}

\author{
Thasni T, C Kalaiarasan, K A Venkatesh
}

\begin{abstract}
Cloud computing allows on-demand access and fast network connection to a shared resource pool. Most companies are switching to Cloud due to the popularity and benefits of using Cloud Services. So finding a suitable and best cloud provider is a challenge for all users. Several ranking methods, such as AHP, TOPSIS, had been suggested to solve this problem by multicriteria decision making techniques. But, many of the works focused on a subset of the main QoS attributes for ranking. Cloud Services Measurement Initiatives Consortium (CSMIC) has released Service Measurement Index attributes for effectively comparing the Cloud services. The comparison of services provided by cloud based on SMI attributes which are qualitative as well as quantitative in nature is studied in this paper by one of the non-parametric methods called Data Envelopment Analysis (DEA) and ranked the cloud services based on the efficiency scores obtained by DEA. The cloud users can select the best suitable Cloud service using the proposed approach that best suit their $Q 0 S$ requirements.
\end{abstract}

Keywords: MCDM, DEA SMI, Cloud Service, QoS, CSMIC.

\section{INTRODUCTION}

Next generation computing platform Cloud computing is growing and emerging rapidly. The Internet provides access to a range of computer resources. The leading companies and individual cloud machines are Amazon, Google, Sales Force and Microsoft. Several examples of common resources can be managed using the network framework and digital services. Cloud-based services concentrate on user computing, software and data. The network should be available for users who want to use cloud services. Cloud users can purchase the services or use the services in the cloud at no cost. Various types of services, including platform as services, infrastructure as services and software as services are available in the cloud. Cloud provides most of the choices such as enabling customers to access the cloud, or providing the consumer with computing power over the cloud, or storing any of their data without worrying about privacy issues , or allowing the consumer to use or use the application for their own use, and enabling the consumer to access the database

Revised Manuscript Received on April 9, 2020.

* Correspondence Author

Thasni T*, Assistant Professor, CSE Department ,School Of Engineering ,Presidency University, Email: thasniumer@gmail.com

C. Kalaiarasan, Associate Dean, School Of Engineering, Presidency University. Email: kalaiarasan@presidencyuniversity.in

K.A.Venkatesh, Professor of Math and Computer Science, Myanmar Institute of Information Technology,73rd Street, Ngu Shwe War, Chanmyathazi Township, Mandalay, Myanmar. Email: ka_venkatesh@miit.edu.mm

(C) The Authors. Published by Blue Eyes Intelligence Engineering and Sciences Publication (BEIESP). This is an open access article under the CC BY-NC-ND license (http://creativecommons.org/licenses/by-nc-nd/4.0/) through the cloud. Most cloud providers based on their resources are almost similar. The choice of the most appropriate cloud provider is a major challenge. Various methods for the ranking of cloud services are suggested by various authors. MCDM methods such as AHP, TOPSIS etc. are some of the most effective techniques. It is necessary to choose a suitable cloud provider accurately in order to increase the level of confidence between customers and suppliers. A collection of Main Performance Indicators (KPIs) for cloud service assessment are given in this context by the Cloud Service Measurement Initiative Consortium (CSMIC) [1]. The selection of a cloud provider is a decision taking challenge requiring several parameters, in which many QoS variables play a significant role in decision making. Therefore, a better MCDM approach may be needed to meet customer requirements and identify the cloud services on the basis of their skill. This paper proposes an approach to choose an ideal cloud service provider based on the QoS attributes of cloud services among different solutions. A different MCDM approach has been established in the recent research, such as AHP [2], Fuzzy AHP with the Delphi method [3] and Fuzzy ELEC TOPSIS [4]. For the evaluation and selection of Cloud Service Provider Selection, Azadi et al proposed a new approach called Data Envelopment Analysis which concentrates on SMI attributes for the ranking of Providers [5].Data Envelopment Analysis is a powerful analytical investigative tool that has been recognized to help realistic decisions. The following parts of this document is structured properly as follows. Part 2 presents a brief overview of the cloud service selection related works. Section 3 defines the evaluation criteria required for the selection of cloud service providers, Section 4 describes with its key elements the proposed ranking approach. Section 5 gives the ranking results and discussions followed by section 6 which concludes with future work

\section{RELATED WORK}

Garg et al. [6] developed a system using the AHP as a cloud service provider rating system in confidence in attribute weights measurement. The authors calculated the QoS requirements of the Cloud- Service- Measurement- Initiative -Consortium (CSMIC) and used key indicators of performance (KPIs) to assess the service of the cloud. Nonetheless, selection of the service and rating of the same are based solely on CSMIC's quantifiable criteria and does not recognize the attributes which are not quantifiable for QoS trustworthiness CSP selection. The researcher did not take into account in this paper, the incoherence with the evaluation criteria in the issue of selection of cloud service. $\mathrm{R}$.

Published By:

Blue Eyes Intelligence Engineering

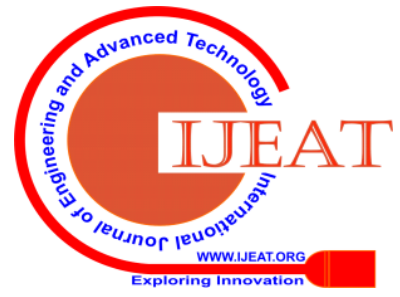


Ranjan Kumar et al [7] explains about the importance of best service among the available services in the cloud and showed the previous approaches and limitations of those methods and he proposed a new Computational Framework by combining the TOPSIS and AHP approach to select the best Service for the Customer. A different MCDM approach has been established in the recent research, such as AHP [2], Fuzzy AHP with the Delphi method [3] and Fuzzy ELEC TOPSIS [4]. Performance testing and choosing the right cloud service providers (CSPs) based on service quality (QoS) criteria is one of the greatest obstacles of utilizing cloud computing platforms (Duan, 2017). There are relatively few work initiatives in cloud systems on the website of DEA. Kumar [8] suggested the tool to test cloud services based on DEA, AHP and order choice techniques in a similar way to TOPSIS (Topsis), based on cloud services. In order to determine the relative productivity of Iaas programs, Wang [9] submitted a non-parametric DEA. This definition is focused on basic requirements such as storage, memories and CPU [9]. This framework includes cloud resources. Filio- poulou et al. [10] proposed a DEA input-oriented model for cloud services output measurement focused on functional and non-functional parameters such as reliability. In paper [5] authors suggested a network DEA for the efficiency calculation of CSPs to resolve the above shortcoming. Authors have used the QoS attributes like Latency, Availability, Memory, CPU, Data transfer etc for comparing the efficiency of CSPs. In analyzing network measurements, a wider overview was given where divisional performance was expressed in the overall output figures. This lets corporate administrators and decision-makers rely on cloud infrastructure correctly.

\section{CRITERIA FOR RANKING}

In compliance with the International Organization for Standardization (ISO) specifications, SMI attributes have been defined in CSMIC [2]. Significant business performance indicators (KPIs) that provide an institutionalized system for measuring and evaluating market advantage. The customer will be able to choose an acceptable cloud provider that meets the efficiency, security, privacy, utilizability and QoS requirements based on the SMI (Service Measurement Index) attributes.

The following features are considered for the evaluation of the Cloud services. In [5], authors have considered only the performance related quantitative attributes of the CSPs .but this paper addresses other important SMI attributes for the comparison and evaluation of Cloud Service Providers.

\section{A. Agility}

The most essential preferred role in Cloud registration is to make an organization more accessible. This relationship may be quickly expanded and modified. New technologies should be implemented in IT depending on the needs of the client. Agility, as the improvement metric variable indicating the same, is calculated in SMI. The company must test whether the resources offered by the Cloud supplier are scalable and adaptable and if they are versatile, taking into consideration the Flexibility aspect from one vendor to another. Saurabh
Garg [6] was also used to measure RSRV using the formula mentioned above to rate the providers of cloud services.

\section{B. Response Time}

This measure is used when the job in the virtual environment is demanded and when the job request in the virtual environment is done. This metric provides an image of the cloud's efficiency.

\section{Security and Privacy}

Privacy and security of data are vital issues in almost every environment. Facilitating information in numerous partnerships is a reliable core problem which calls for stringent protection strategies used by cloud providers. For example, the majority of financial services need highly accuracy tests, including reliability and protection of details. In addition, privacy and security are multi-dimensional and include various attributes, including security, respectability and misfortune in details.

\section{Usability}

The ease with cloud providers when using every cloud service is quite significant. The ease of use of a Cloud administration that depend on several variables, such as usability, Installability, learning, operability, ultimately cloud providers which provide different services will comply with QoS criteria defined by CSMIC and thus improve the trust of the cloud provider. This facility would enable more consumers to migrate into the Cloud provider.

\section{E. Availability}

Giving cloud services a higher degree of availability was one of the biggest challenges According to [11] the availability is measured as the percentage of time an application has, despite a fixed time period, and its services are available.Cost

Published By:

Blue Eyes Intelligence Engineering 
The expense of running the cloud for every customer calculates this parameter. The continued expense of all analysis, servicing, contract administration, customer care and accounting procedures, and logistical infrastructure and systems available to maintain a company, including complete loading of the cost of labor typically estimated by actual client at an average unit rate.

\section{PROPOSED CLOUD SERVICE SELECTION APPROACH}

Data Envelopment Analysis [DEA] is indeed a tool for the decision making of a set of comparative units centered on a linear model to calculate the relative efficiencies. DEA is created by Charnes, Cooper, Rhodes (1978).DEA is a linear programming technique designed to calculate the relative performance of operational units with the same aims and objectives. DEA allows for the simultaneous evaluation of several inputs - outputs without the assumption of data distribution. Performance is determined in each case by the inputs or outputs ' proportional change. This DEA model is divided into an input-based model that decreases input meeting the specified output rates, and also an output-based model which increases outputs while demanding more than any observable input values.

And also DEA models are subdivided by incorporating weight restrictions in terms of returns to scale. Charnes, Cooper, and Rhodes (1978) initially implemented the performance measurement of the DMUs for constant scale returns (CRS), where all DMUs run at their optimum scale.

Consider nd decision making units $D M U_{j}(\mathrm{j}=1, \ldots$, nd)approved , which is intended to be used to transform md inputs $s_{i j}\left(\mathrm{i}=1, \ldots\right.$, md) to l outputs $t_{r j}(\mathrm{r}=1$, . v. . , l) and to allow DMU0 as the DMU to be evaluated. Suppose the weights of input and output are positive quantities. By assuming that all DMUs have efficiency value which will be less than or equal to 1, efficiency of DMU0 which is $\theta 0$ should be calculated. Efficiency value of DMUs is called the ratio of weighted number of outcomes to the weighted number of inputs. Therefore, inputs and outputs are given separately for pi $(i=1, . . . \mathrm{md})$ and $\mathrm{qr}(\mathrm{r}=1, \ldots ., \mathrm{s})$ weights and the following equations are used for calculating the efficiency of these DMUs.

\section{- Objective function:}

$\operatorname{Max} \theta_{0}=$

$$
\frac{q_{1} t_{10}+q_{2} t_{20}+\cdots+q_{s} t_{s 0}}{p_{1} s_{10}+p_{2} s_{20}+\cdots+p_{m d} s_{m d 0}}
$$

- Subject to constraints : $\frac{q_{1} t_{1 j}+q_{2} t_{2 j}+\cdots+q_{s} t_{s j}}{p_{1} s_{1 j}+p_{2} s_{2 j}+\cdots+p_{m d} s_{m d j}} \leq 1(j=1,2,3, \ldots \ldots n d)$

$$
\mathrm{q}_{1}, \mathrm{q}_{2}, \mathrm{q}_{3}, \mathrm{q}_{4}, \ldots . \mathrm{q}_{\mathrm{s}} \geq 0
$$$$
\mathrm{p}_{1}, \mathrm{p}_{2}, \mathrm{p}_{3} \ldots \ldots \ldots ., \mathrm{p}_{\mathrm{md}} \geq 0
$$

The fractional model (1) is translated into linear programming problem model in order to solve this problem, assuming the cumulative weighted input of that particular DMU equals unity

\section{- Objective function:}

$\operatorname{Max} \theta_{0}=\mathrm{q}_{1} \mathrm{t}_{10}+\mathrm{q}_{2} t_{20}+\cdots+\mathrm{q}_{\mathrm{s}} \mathrm{t}_{\mathrm{s} 0}$

- Subject to constraints :

$$
\begin{aligned}
& \mathrm{p}_{1} \mathrm{~s}_{1 \mathrm{j}}+\mathrm{p}_{1} \mathrm{~s}_{1 \mathrm{j}}+\cdots+\mathrm{p}_{\mathrm{md}} \mathrm{s}_{\text {mdj }}=1 \\
& \left(\mathrm{q}_{1} \mathrm{t}_{1 \mathrm{j}}+\mathrm{q}_{2} \mathrm{t}_{2 \mathrm{j}}+\cdots+\mathrm{q}_{\mathrm{s}} \mathrm{t}_{\mathrm{sj}}\right)- \\
& \left(\mathrm{p}_{1} \mathrm{~s}_{1 \mathrm{j}}+\mathrm{p}_{1} \mathrm{~s}_{2 \mathrm{j}}+\cdots+\mathrm{p}_{\mathrm{md}} \mathrm{s}_{\mathrm{mdj}}\right) \leq 0 \\
& \mathrm{q}_{1}, \mathrm{q}_{2}, \mathrm{q}_{3}, \mathrm{q}_{4,}, \ldots \mathrm{q}_{\mathrm{s}} \geq 0 \\
& \mathrm{p}_{1}, \mathrm{p}_{2}, \mathrm{p}_{3}, \mathrm{p}_{4} \ldots \ldots \mathrm{p}_{\mathrm{md}} \geq 0
\end{aligned}
$$

If it is found that a DMU is ineffective, a list of other productive DMUs may be used as a comparison for this DMU and then generate a more influential output with an analogous input aggregate or use fewer to achieve a comparable performance combination or a mixture of the two so as to improve the efficiency of that DMU. In order to achieve various output scores, the DEA requirement that the performance of the DMU is lower or equivalent to the unity is omitted that allows to gain an output score that is higher than one for an exceptionally effective DMU, so this time the importance of performance is specific for all DMUs and now they are conveniently categorized.. Table 1 shows SMI attributes which are identified as the input and output parameters of CSPs for DEA formulation. Based on the requirements, DEA can be input based or output based with any of the returns to scale. CCR DEA is a well-known basic model in DEA. The fundamental concept of this model is to construct a piece-by-piece linear convex boundary such that the boundary covers the expected input and output data as per the defined goal function. Efficiency values are determined by a series of linear programs from the linear boundaries created. The DMU is the entity that can turn inputs into outputs. It's also the decision-making center. Assume we have $n$ entities that generate $\mathrm{n}$ separate outputs from $\mathrm{m}$ separate inputs. The relative efficiency of all DMUs in question must be calculated. The case is modeled as a continuous fraction of the situation is designed as a fractional linear program aiming at maximizing the ratio between the sum of the weighted output and the sum of the weighted inputs subject to non-decreasing weight restrictions. Assuming that the amount is maximized, the proportional value should be at most 1 . This implies finding the right weights to optimize performance. The orientation, input orientation or output orientation will be addressed as another significant topic in the DEA method. The calculation of efficiency increases productivity by reducing the input quantities while calculating the functional value, while the output numbers remain unaltered. This method is focused on the functional utility of the expense limit. The outgoing orientation is simply to reverse the input guideline that increases the efficiency rating by reducing the output quantity in a relative manner, without altering the input quantity [12].

\section{- CCR Efficiency}

CCR efficiency: if $\theta=1$, then a DMU is efficient in the context of CCR, that means, for non-negative $p$ and $q$, at least one optimal solution exits , if not the DMU is not efficient. In Linear programming problems, duality has as important role. Assume there are k DMUs, which uses md inputs to generate nd outputs. The dual of the model (2) can be expressed for a real number $\theta^{*}$ and a non-negative vector $\lambda=\left(\lambda_{1}, \lambda_{2}, \ldots, \lambda_{\mathrm{k}}\right)$ as

$$
\theta^{*}=\operatorname{mini} \theta
$$

Published By:

Blue Eyes Intelligence Engineering

\& Sciences Publication

(C) Copyright: All rights reserved. 
Subject to

$$
\begin{array}{ll}
\sum_{j=1}^{k} s_{i j} \lambda_{j} \leq \theta s_{i 0} & 1 \leq \mathrm{i} \leq \mathrm{nd} \\
\sum_{j=1}^{k} t_{r j} \lambda_{j} \leq t_{i 0} & 1 \leq \mathrm{r} \leq \mathrm{md} \\
\lambda_{j} \geq 0, \forall j &
\end{array}
$$

If $\theta^{*}=1, \lambda_{\mathrm{j}}{ }^{*}=1$ and $\lambda_{0}=0$, for $\mathrm{j} \neq 0$,then the dual model (3) is feasible. This step should be repeated as many times as the number of DMUs. Note that on the border points, optimal solutions are found and not every border point is efficient. We use the modified model (4) in such a scenario.

Subject to:

$$
\operatorname{Max} \sum_{i=1}^{n d} s l_{i}^{-}+\sum_{r=1}^{m d} s l_{r}^{+}
$$

$$
\begin{gathered}
\sum_{j=1}^{k} s_{i j} \lambda_{j}+s l_{i}^{-}=\theta s_{i 0} \quad 1 \leq \mathrm{i} \leq \mathrm{nd} \\
\sum_{j=1}^{k} t_{r j} \lambda_{j}-s l_{r}^{+}=t_{i 0} \quad 1 \leq \mathrm{r} \leq \mathrm{md} \\
\lambda_{j} \geq 0 \quad 1 \leq j \leq k \\
s l_{i}^{+} \geq 0 \quad 1 \leq i \leq n d
\end{gathered}
$$

Where $s l_{r}^{-}$and $s l_{i}^{+}$are slack variables.
DEA efficiency: Any DMU is efficient if and only if $\theta^{*}=1$ and all slack variables are zero. In this work, we have proposed CCR- Input oriented DEA to compute the efficiency scores and ranked the DMU's based on these efficiency scores.

\section{RESULT AND DISCUSSION}

$\mathrm{R}$ is an open source tool for statistical computing. Because of its capabilities, it is a popular and powerful tool. R tool is

\begin{tabular}{|c|c|c|c|c|c|c|}
\hline Summary Statistics & $\begin{array}{c}\text { Response } \\
\text { Time }\end{array}$ & Cost & Security & Availability & Usability & Agility \\
\hline Mean & 360.7417 & 192.8667 & 34.66667 & 99.412133 & 32.66667 & 30.66667 \\
\hline Standard Error & 114.4689 & 10.95044 & 2.983993 & 0.3296771 & 2.991687 & 2.953017 \\
\hline Median & 135.39 & 194.5 & 30 & 99.875 & 30 & 30 \\
\hline Mode & \#N/A & 165 & 50 & 99.5 & 50 & 30 \\
\hline Standard Deviation & 626.9719 & 59.978 & 16.344 & 1.8057159 & 16.38614 & 16.17434 \\
\hline Sample Variance & 393093.8 & 3597.361 & 267.1264 & 3.26061 & 268.5057 & 261.6092 \\
\hline Kurtosis & 18.11649 & -1.19055 & -1.33419 & 27.982084 & -1.45717 & -1.45376 \\
\hline Skewness & 4.053224 & -0.16883 & -0.46968 & -5.214884 & -0.25916 & -0.06271 \\
\hline Range & 3271.4 & 199 & 40 & 9.999 & 40 & 40 \\
\hline Minimum & 50 & 86 & 10 & 90 & 10 & 10 \\
\hline Maximum & 3321.4 & 285 & 50 & 99.999 & 50 & 50 \\
\hline Sum & 10822.25 & 5786 & 1040 & 2982.364 & 980 & 920 \\
\hline Count & 30 & 30 & 30 & 30 & 30 & 30 \\
\hline
\end{tabular}
used for implementing our DEA model, which is input oriented and Constant Return Scale type. R 3.6.3 version was used for the implementation. Data set used in this study was collected from various sources which are available online. We considered 3 inputs and 3 outputs as shown in table I.

Table I: Input and Output Variables

\begin{tabular}{|r|l|l|}
\hline \multicolumn{1}{|c|}{ Sl No } & Input Variables & Output Variables \\
\hline 1 & Response Time & Availability \\
\hline 2 & Cost & Agility \\
\hline 3 & Security and Privacy & Usability \\
\hline
\end{tabular}

TABLE II

We have considered 30 cloud service providers for this study. DEA is applied to obtain the efficiency scores of the cloud service providers. The input and out variables considered for this study are given in Table I. Based on the efficiency score, the cloud services providers are ranked. The three SMI attributes selected as input variables are response time, cost and security. The output variables selected from the SMI attributes are availability, usability and agility. The SMI attributes are the standard QoS attributes which help us to compare the performance of the services of the cloud. Many online platforms such as cloud Harmony, Armor provide the performance details of cloud services performance to benchmark these. The input and output variables such as response time, cost, security, availability, usability and Agility are well explained in the above section. Table II gives

the summary statistics of the input and output variables in our study. Summary StatisticsThe efficiency scores of 30 cloud service providers that means 30 DMUs are computed using Constant Returns Scale and presented in table III. The DMUS are ranked based on the efficiency scores obtained by DEA. It is clear from the table that 10 DMUS are $100 \%$ efficient and DMU23 is the least efficient service.

\& Sciences Publication

(C) Copyright: All rights reserved. 
TABLE III

\begin{tabular}{|c|c|c|c|c|c|}
\hline DMU & Efficiency & Rank & DMU & Efficiency & Rank \\
\hline DMU5 & 1 & 1 & DMU3 & 0.794 & 16 \\
\hline DMU8 & 1 & 1 & DMU14 & 0.783 & 17 \\
\hline DMU9 & 1 & 1 & DMU7 & 0.78 & 18 \\
\hline DMU12 & 1 & 1 & DMU10 & 0.765 & 19 \\
\hline DMU15 & 1 & 1 & DMU11 & 0.707 & 20 \\
\hline DMU18 & 1 & 1 & DMU30 & 0.678 & 21 \\
\hline DMU20 & 1 & 1 & DMU26 & 0.677 & 22 \\
\hline DMU21 & 1 & 1 & DMU16 & 0.674 & 23 \\
\hline DMU22 & 1 & 1 & DMU28 & 0.643 & 24 \\
\hline DMU24 & 1 & 1 & DMU2 & 0.605 & 25 \\
\hline DMU19 & 0.999 & 11 & DMU17 & 0.568 & 26 \\
\hline DMU4 & 0.994 & 12 & DMU6 & 0.56 & 27 \\
\hline DMU29 & 0.922 & 13 & DMU1 & 0.559 & 28 \\
\hline DMU25 & 0.809 & 14 & DMU13 & 0.535 & 29 \\
\hline DMU27 & 0.8 & 15 & DMU23 & 0.437 & 30 \\
\hline
\end{tabular}

The non-parametric method known as Data Envelopment Analysis (DEA) has deployed to compute the efficiency scores of the considered DMUs' based on the selected inputs and outputs. The development of DEA literature is incomparable across several other tools. DEA is an extension of linear programming, in which a number of linear programming problems (equal to the number of DMUS) have to be solved to obtain relative efficiency Scores.

The efficiency scores of 30 DMU's has been computed and given in Table II. In this study, we deployed input-oriented CRS model. A DMU with efficiency score 1 is fully efficient. 10 DMUs are fully efficient and they have been ranked number 1.From table II, one could identify which service provider is performing better.

In this article, the selection of suitable cloud service is a decision-making problem addressed. The DEA method used in this paper successfully ranked the services based on the performance. It helps the user to take appropriate decision before selecting a suitable cloud service.Efficiency Scores and ranks of DMUs

The $\mathrm{R}$ tool is very user friendly for generating the required results and plots. R 3.6.3 version was used for the efficiency calculation of DMUs using DEA .The DMUs are then ranked according to the efficiency score values and rank one is given to DMU having efficiency value 1 and the necessary plots are generated using the efficiency values and ranks of all the 30 DMUs.

DMU5 ,8,9,12,15,18,20,21,22 and DMU24 are having efficiency 1 ,so they are ranked as rank 1.Thirty DMUs are used in this study. So in turn R solves 30 Linear Programming Problems to produce efficiency scores .So above mentioned DMUs are fully efficient ones. So users can undoubtedly choose those services.

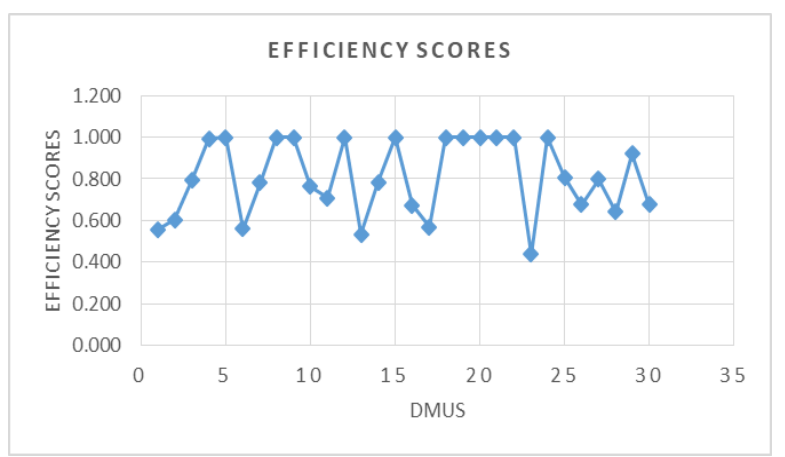

Fig 1: Efficiency scores of DMUs

The efficiency scores of all the 30 DMUs are plotted in the graph below which is shown in figure 1 .

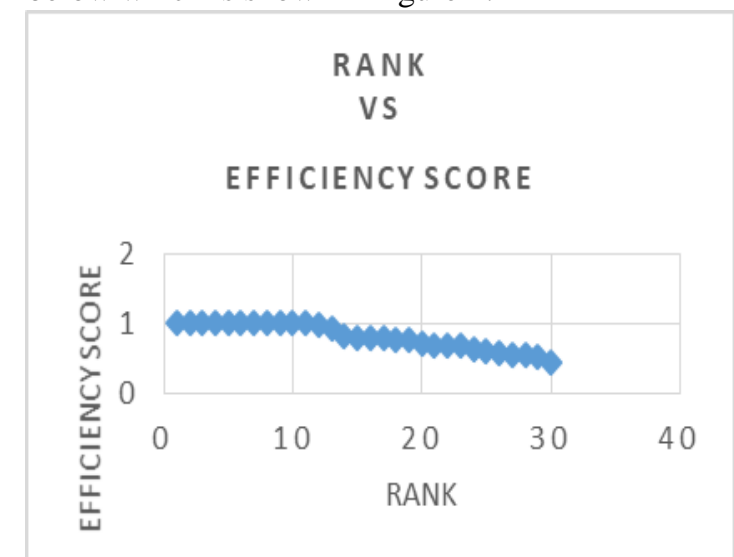

Fig 2: Graph representing Efficiency score versus rank The graphical representation of the relation between efficiency scores and ranks of the DMUs are plotted in the below graph shown in figure 2 .

The ranks of all the 30 DMUs are shown in the below graph in figure 2.DMU5 is the most efficient service with rank 1 and DMU23 is the least efficient one with a rank of 30.

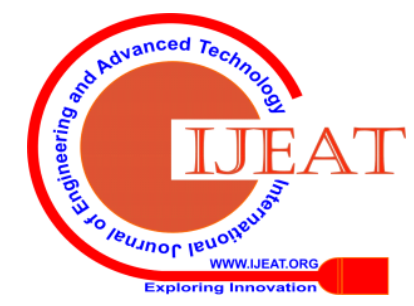




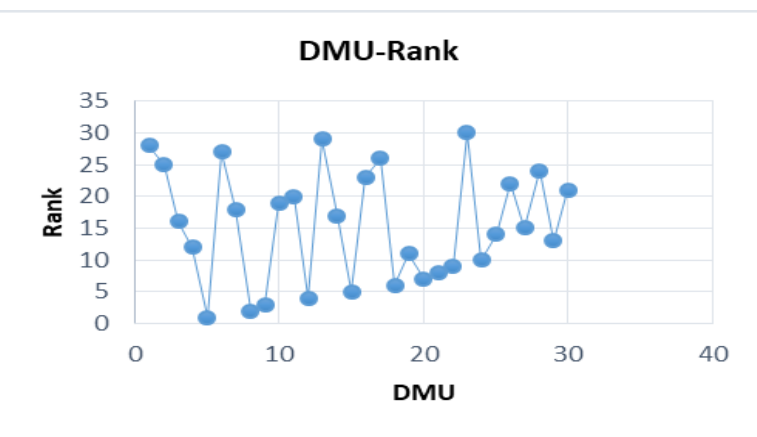

Fig 3. Ranks of 30 DMUs

\section{CONCLUSION}

In a very competitive world, the performance quality of CSP with QoS metrics is one of the big problems for cloud service consumers and providers. Our approach addressed the comparison of Cloud Services based on SMI attributes and considered cloud service providers are ranked based on the computed efficiency scores. This study, certainly help the cloud service users to choose their service providers. The authors are in process of applying other MCDM models to study in order to rank the cloud service providers

\section{REFERENCES}

1. J. Siegel and J. Perdue, "Cloud Services Measures for Global Use: The Service Measurement Index (SMI)," 2012 Annual SRII Global Conference, San Jose, CA, 2012, pp. 411-415.

2. Garg, S.K.; Versteeg, S.; Buyya, R.: A framework for ranking of cloud computing services. Future Gener. Comput. Syst. 29(4), 1012-1023 (2013)

3. Liu, S.; Chan, F.T.; Ran, W.: Decision making for the selection of cloud vendor: an improved approach under group decision-making with integrated weights and objective/subjective attributes. Expert Syst. Appl. 55, 37-47 (2016)

4. Lee, S.; Seo, K.-K.:A hybrid multi-criteria decision-making model for a cloud service selection problem using BSC, fuzzy Delphi method and fuzzyAHP.Wirel. Pers. Commun. 86(1), 57-75 (2016)

5. Azadi, M., Emrouznejad, A., Ramezani, F., \& Hussain, F. K. (2019). Efficiency measurement of cloud service providers using network data envelopment analysis. IEEE Transactions on Cloud Computing, 1-1.

6. Garg, S.K.; Versteeg, S.; Buyya, R.: A framework for ranking of cloud computing services. Future Gener. Comput. Syst. 29(4), 1012-1023 (2013)

7. Rakesh Ranjan Kumar, Siba Mishra, Chiranjeev Kumar,: A Novel Framework for Cloud Service Evaluation and Selection Using Hybrid MCDM Methods, Arab J Sci Eng,Springer , 2017

8. S. Kumar, "Evaluating cloud services based on DEA, AHP and 551 TOPSIS," D.G.R. Gangadharan, Institute of Development and 552 Research in Banking Technology, Hyderabad, 2014.

9. C. Xu, Y. Ma, and X. Wang, "A non-parametric data envelopment 605 analysis approach for cloud services evaluation," in Proc. Serv.- 606 Oriented Comput., 2015, pp. 250-255

10. E. Filiopoulou, P. Mitropoulou, N. Lionis, and C. Michalakelis, 526 "On the efficiency of cloud providers: A DEA approach incor527 porating categorical variables," IEEE Trans. Cloud Comput., 2018, 528 in press, doi: 10.1109/TCC.2018.2850889

11. Toeroe M, Tam F (2012) Service Availability: Principles and Practice. JohnWiley\&Sons.http://www.wiley.com/WileyCDA/WileyTitle/prod uctCd-1119954088.html.

12. K A Venkatesh and N Pushkala(2019), Performance evaluation and measures (Chapter 10 ), Modern Optimization Methods for Science, EngineeringandTechnology,https://iopscience.iop.org/book/978-0-75 03-2404-5/chapter/bk978-0-7503-2404-5ch10

13. Venkatesh K A, Pushkala N and Mahamayi J 2017 IFA - Off-Balance Sheet Items and Performance Evaluation of Public and Private sector banks in India: A DEA approach CFA Institute https://arx.cfa/post/IFA-Off-Balance-Sheet-Items-and-Performance-E doi:10.1109/tcc.2019.2927340

valuationof-Public-and-Private-sector-banks-in-India-A-DEA-approa ch-4968.html

14. Cooper W, Seiford L M and Tone K 2007 Data Envelopment Analysis: A Comprehensive Text with Models, Applications, References and DEA-Solver Software (Berlin: Springer)

15. E. Filiopoulou, P. Mitropoulou, N. Lionis, and C. Michalakelis,526 "On the efficiency of cloud providers: A DEA approach incor-527 porating categorical variables," IEEE Trans. Cloud Comput., 2018,528 in press, doi: 10.1109/TCC.2018.2850889

16. S. K. Garag, S. Verstegg and R. Buyya, "A framework for ranking of cloud computing services," Future Generation Comput. Syst., vol. 29, no. 4, pp. 1012-1023, 2013

17. N. Ghosh, S. K. Ghosh, and S. K. Das, "SelCSP:Aframework to facilitate selection of cloud service providers," IEEE Trans. Cloud Com-534 put., vol. 3, no. 1, pp. 66-79, 2014. doi: 10.1109/TCC.2014.2328578

18. S. Kumar, "Evaluating cloud services based on DEA, AHP and TOPSIS," D.G.R. Gangadharan, Institute of Development and Research in Banking Technology, Hyderabad, 2014

19. M. Supriya, K. Sangeeta, and G. K. Patra, "Trustworthy cloud service provider selection using multi criteria decision making method," Eng. Letts., vol. 24, no. 1, pp. 1-10, 2016.

20. I.Widiarto, A. Emrouznejad, and L. Anastasakis, "Observing choice of loan methods in not-for-profit microfinance using data envelopment analysis," Expert Syst.Appl., vol. 82, pp. 278-290, 2017.

\section{AUTHORS PROFILE}

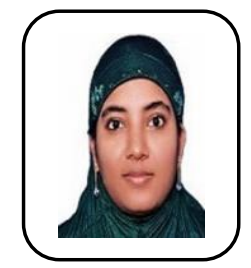

Thasni $\mathbf{T}$ is currently working as Assistant Professor in Computer Science Department in Presidency University,Bangalore and also pursuing $\mathrm{PhD}$ in Presidency University. She is having 3 years of teaching experience and she did her masters in Computer and Information Science in CUSAT University . She completed her Bachelor of Technology in Computer Science and Engineering in MG University. Her research interests include Cloud Computing ,Artificial Intelligence ,Data Analytics ,Data Mining, Machine Learning, IoT and Neural Networks.

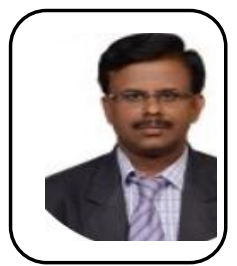

Dr.C. Kalaiarasan pursued his B.E. degree in Computer Science and Engineering from Bharathidasan University in the year 1989 M.S.,degree in Software Systems from Birla Institute of Technology and Science, Pilani. M.E., degree in Computer Science and Engineering from PSG college of Technology and PhD Computer Science and Engineering from Anna University, Chennai. He has been in the field of teaching since 25 years and he also gained an experience of 5 Years in Industry. He was a Professor, Department of Computer Science and Engineering at PSG College of Technology Coimbatore from the year 2000 to 2010. He was the Principal, Tamilnadu College of Engineering (TCE) Coimbatore.He also served as Principal and Dean at SNS Technical Institutions, Coimbatore from 2015 to 2018. Currently he is working as Associate Dean, School of Engineering, Presidency University, Bengaluru. He has published more than 50 papers in international and national journals and conference proceedings. His research area includes Computer Networks, TCP/IP, Wireless Networking, Software Engineering, IoT and Database Systems.

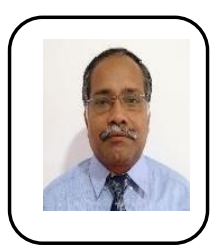

Dr. K A Venkatesh has 3 decades of teaching experience and 4 years of Industry experience, served as CTO. He served as Registrar, Dean- Research, Head of Department, Principal. He has 65 publications in journal, conference proceedings, study materials and coauthored a book on Discrete Mathematics. He has completed his Ph.D from Alagappa University. Also authored 5 book chapters. He is a member of Systems Society of India, RMS, Constrained Programming Association USA, Academy of Discrete Mathematics and Applications. Serving as Adjunct Professor at IIIT-B and Professor of Math and Computer Science,

Myanmar Institute of Information Technology, Mandalay, Myanmar. His area of research interests are Algorithm, Benchmarking, Combinatorial Optimization and Banking \& Finance. 